УДК 001.89, 004.94, 005.8

DOI 10.52575/2687-0932-2021-48-3-552-563

\title{
Системная динамика в управлении междисциплинарными проектами
}

\author{
Маслобоев А.В., Путилов В.А. \\ Институт информатики и математического моделирования Федерального исследовательского центра \\ «Кольский научный центр Российской академии наук», \\ Россия, 184209, Мурманская область, г. Апатиты, ул. Ферсмана, 14 \\ E-mail: masloboev@iimm.ru
}

\begin{abstract}
Аннотация
В работе рассматриваются вопросы применения метода системной динамики для анализа и моделирования процессов управления комплексными междисциплинарными проектами и программами. Проведен анализ базовых структурных элементов проектного управления и причинно-следственных связей, моделирующих задержки, обратные связи и нелинейные взаимозависимости в процессе реализации междисциплинарных проектов и разработок. Предложена технология использования системно-динамических моделей для задач управления научно-исследовательскими проектами, обеспечивающая концептуальный анализ динамической структуры проектов и моделирование динамики их поведения с целью синтеза и оценки сценариев достижения заданных показателей эффективности в условиях временных и ресурсных ограничений. Результаты исследования смогут найти применение для улучшения существующей практики управления проектами различного масштаба в научно-инновационной, образовательной, инвестиционной и других сферах.
\end{abstract}

Ключевые слова: системная динамика, управление проектами, системно-динамическая модель, междисциплинарные исследования и разработки, концептуальное моделирование.

Благодарности: результаты получены в рамках выполнения государственного задания ИИММ КНЦ РAH (№ 0226-2019-0035).

Для цитирования: Маслобоев А.В., Путилов В.А. 2021. Системная динамика в управлении междисциплинарными проектами. Экономика. Информатика, 48 (3): 552-563. DOI 10.52575/26870932-2021-48-3-552-563.

\section{System dynamics for management of interdisciplinary projects}

\author{
Andrey V. Masloboev, Vladimir A. Putilov \\ Institute for Informatics and Mathematical Modeling of the Federal Research Centre «Kola Science Centre \\ of the Russian Academy of Sciences», \\ 14 Fersman St, Apatity, Murmansk region, 184209, Russia \\ E-mail: masloboev@iimm.ru
}

\begin{abstract}
The study considers application issues of system dynamics method for the purpose of analysis and modeling of the comprehensive interdisciplinary project and research program management processes. The analysis of basic structure elements of the project management and cause-effect relations, which simulate delays, feedbacks and non-linear interdependencies during the process of interdisciplinary project and development implementation, is carried out. The technology for use of system-dynamic models within the bounds of management problem-solving of research and development projects, which provides conceptual analysis of project dynamic structure and modeling of project conduct dynamics purposely to scenarios synthesis and assessment of the given efficiency index values achievement under temporal and resource restrictions, has been proposed. The results of investigation can be applicable for improvement of the existing practice of project management of the various scales in research, innovation, education, investment and other areas.
\end{abstract}

Keywords: system dynamics, project management, system-dynamic model, interdisciplinary research and developments, conceptual modeling. 
Acknowledgements: the research results were obtained within the framework of the State Research Program of the Institute for Informatics and Mathematical Modeling of the Kola Science Centre of RAS (project No. 0226-2019-0035).

For citation: Masloboev A.V., Putilov V.A. 2021. System dynamics for management of interdisciplinary projects. Economics. Information technologies, 48 (3): 552-563 (in Russian). DOI 10.52575/2687-0932-202148-3-552-563.

\section{Введение}

На сегодняшний день в условиях бурного развития цифровой экономики, научной дипломатии и международного технологического противоборства возрастают требования к качеству научных исследований и разработок и эффективности управления связанными с этой сферой сложными процессами. На практике эти требования определяются общими и специфическими аспектами социально-экономического и общественно-политического характера, имеющими особое значение для текущего развития страны и стратегических задач государственного управления. Современная наука характеризуется конвергенцией и синергизмом, междисциплинарностью и неопределенностью, возрастающими объемами разноплановой информации, требующими обработки и анализа для получения новых и полезных знаний. Комбинация этих свойств приводит к увеличению сложности процессов научных исследований и разработок, что обусловливает необходимость в создании новых форм и инструментов организационного управления этими процессами. В связи с этим одной из важных и наиболее актуальных проблем в этой области является планирование и реализация междисциплинарных проектов и программ исследований, направленных на комплексное изучение различных аспектов функционирования сложных объектов, процессов и систем различной природы и масштаба, а также закономерностей и тенденций их поведения и развития с учетом пространственно-временной когерентности и параметрической согласованности конкретных наблюдений и измерений.

Для решения этой проблемы на практике традиционно применяются различные методы исследований, как при построении моделей изучаемых объектов и явлений, так и при организации экспериментальных исследований параметров этих объектов и явлений. Эти методы предполагают интеграцию локальных моделей в глобальные, объединение локальных исследований в глобальные программы, привлечение специалистов из разных областей знаний. Построению моделей управления комплексными междисциплинарными проектами в последнее десятилетие уделяется серьезное внимание. Например, в работах [Игнатьев и др., 1986; Кузьмин и др., 1991; Олейник, Ломов, 2016; Маслобоев, Путилов, 2016; Быстров, Маслобоев, 2018; Олейник, Фридман, 2020] рассматриваются подходы и методы управления научными исследованиями, основанные на разработке и формальном анализе концептуальных и онтологических моделей научно-исследовательских задач. Эти модели достаточно адекватно отражают структуру процесса научных исследований с необходимой степенью полноты и непротиворечивости, но не динамику этого процесса, поскольку в большинстве своем представляют собой замкнутые статические конструкции. Для изучения динамических характеристик процессов междисциплинарных исследований, наряду с известными и апробированными методами концептуального анализа, на практике необходимо также применять специальные методы, к которым относится метод системной динамики [Forrester, 2007a; Forrester, 2007b], предложенный в 50-х годах Дж. Форрестером.

В последнее время применение метода системной динамики в задачах организации и планирования научных исследований и разработок вновь оживилось и расширилось до управления междисциплинарными проектами различного уровня и масштаба - от науки и образования до крупного бизнеса, государственного управления и цифровой экономики. В управлении проектами роль системной динамики заключается в информационноаналитической поддержке (сценарном анализе, прогнозировании, оценке, планировании) 
процессов развития и реализации проектов на всех стадиях их жизненного цикла. Модели системной динамики могут использоваться также для оптимизации и реинжиниринга уже реализуемых проектов, согласования интересов и разрешения конфликтных ситуаций внутри проектных групп, обучения исполнителей, создания автоматизированных средств анализа и тестирования планируемых проектов и т. д. В настоящей работе анализируются основные достижения в области управления проектами на основе метода системной динамики, основные структуры, действующие в проектах. Кроме того, рассматривается, каким образом системнодинамические модели, реализованные на компьютере, могут использоваться для стратегического планирования проектов. Предлагаются направления практического применения системной динамики в управлении междисциплинарными проектами.

\section{Постановка задачи}

Управление междисциплинарными проектами [Новиков, 2007; Маслобоев, 2019] сложная динамическая задача. Известные статические модели и методы, такие как программно-целевое планирование, функционально-целевой подход, недостаточно полно описывают динамику внутренней структуры жизненного цикла междисциплинарных проектов, определяющей характер поведения проекта и его развитие во времени. Однако в практике управления проектами для получения максимальных эффектов важное значение имеют анализ и моделирование именно динамических характеристик процессов развития проекта - от разработки, планирования и реализации до контроля исполнения, оценки результатов и реорганизации. В рамках данной работы выделяются общие и специальные компоненты динамической структуры междисциплинарных проектов и определяется, как эти компоненты могут быть использованы для понимания динамики поведения проектов. Эффективное управление является критическим фактором для успешного развития междисциплинарных проектов. Однако многие проекты терпят неудачи в достижении поставленных целей. Это связано, прежде всего, с тем, что по своей структуре управление междисциплинарными проектами многофункционально и при реализации этого процесса возникают трудности учета всего множества динамических характеристик самого проекта и отдельных этапов его жизненного цикла. К этим характеристикам относятся, например, нелинейные взаимозависимости, обратные связи, запаздывание (задержки) и другие. Еще одной особенностью управления многофазными проектами является необходимость учета и определения взаимосвязей между компонентами динамической структуры проекта и его поведением в процессе развития, характеризующих поведение проекта в зависимости от характера функционирования элементов структуры и влияние формы поведения проекта на доминирование и взаимодействие различных элементов структуры. Исключение этих взаимосвязей из рассмотрения приводит к снижению качества принимаемых решений по управлению проектом и, соответственно, неадекватному развитию проекта.

Эффективность реализации любого проекта и управление его развитием зависят от множества характеристик, включая целеполагание, структуру управляющего цикла, плана поэтапного выполнения работ, способов организации процессов, объемов необходимых ресурсов и затрат на достижение поставленных целей и т. д. Процесс разработки проекта предполагает концептуальное описание всех этапов жизненного цикла проекта и потоков работ (заданий) в рамках этих этапов и между ними. Это описание содержит подробные качественные и количественные характеристики процесса разработки - от этапов постановки задач, определения наличия доступных исполнительных ресурсов и выполнения необходимых итераций внутри этапов и между ними до идентификации задержек в подпроцессах, оптимального распределения ресурсов и распознавания ошибок. Ресурсы характеризуются их количеством (объемом) и эффективностью (производительностью). Эти характеристики, как правило, ограничивают скорость разработки проекта. Размеры (масштаб) проекта определяются количеством задач (заданий), которые необходимо решить в ходе реализации проекта. Задачи проекта описывают условия (например, срок завершения, целевые показатели качества реализации отдельных этапов работ, ожидаемые результаты и т. д.) для финализации проекта. Данные динамические компоненты (задачи, целевые установки, объем работ, масштаб, процесс 
реализации проекта, различные виды требуемых ресурсов) активно взаимодействуют друг с другом в структуре управления проектом и напрямую влияют на ход его выполнения на всех стадиях жизненного цикла проекта. Даже без углубления в детали можно видеть сложность и глобальность задачи управления междисциплинарными проектами. Эта глобальность может быть помехой в прогнозе и анализе [Путилов, Горохов, 2002] и объясняет недостаточную эффективность в принятии решений по управлению такими проектами.

Для исследования внутренней динамики процесса разработки междисциплинарных проектов необходимо системно-динамическое описание причинно-следственных связей, определяющих характер поведения проектов при различных условиях их реализации. Процесс разработки междисциплинарных проектов влияет на характеристики исполнения задач этих проектов ограничением максимально возможной скорости выполняемых работ, зависимостями различных видов исследовательской и другой деятельности и влияниями взаимосвязей между этими задачами. Общая техника сетевого планирования расписания и управления сроками проектов, используемая в методах критического маршрута СРM (Critical Path Method) и оценки проектов PERT (Project Evaluation and Review Technique), моделирует процесс реализации междисциплинарного проекта посредством оценивания длительности выполнения задач и анализа различных внутренних и внешних ограничений на управление проектом между этапами жизненного цикла проекта. При этом требуется поддержание достаточно высокой степени агрегирования динамических характеристик проекта (задач, процессов, ресурсов, размеров). Однако методы CPM и PERT не позволяют явно моделировать динамику итеративных процессов и задач внутри этапов жизненного цикла проекта. Эти методы ориентированы на учет только линейных отношений между элементами процессов разработки и реализации проекта, в то время как большинство взаимосвязей являются нелинейными. Для нивелирования этих недостатков в практике моделирования процессов разработки и управления проектами может быть использован метод системной динамики [Медоуз и др., 2007]. Системно-динамические модели базируются на структуре причинно-следственных связей моделируемых процессов, что позволяет явно и с высокой степенью адекватности описывать и учитывать нелинейные взаимозависимости и задержки в системах управления сложными объектами, а также обратные связи и флуктуации в контуре управления междисциплинарными проектами.

На рис. 1 показаны общие функциональные компоненты, обычно встречающиеся в структуре управления проектами различного типа, взаимозависимости и взаимодействия между этими компонентами. Структура управления проектом включает четыре ключевых функциональных компонента: процесс разработки, управление ресурсами, планирование развития и контроль исполнения. Взаимодействие между этими компонентами основано на обмене информацией и управляющими сигналами. Информационные потоки содержат сведения о требуемых ресурсах, наличии и доступности ресурсов в заданный момент времени, незавершенных и планируемых задачах (работах), статусах развития проекта, конечных сроках реализации проекта или отдельных этапов его жизненного цикла и т. п.

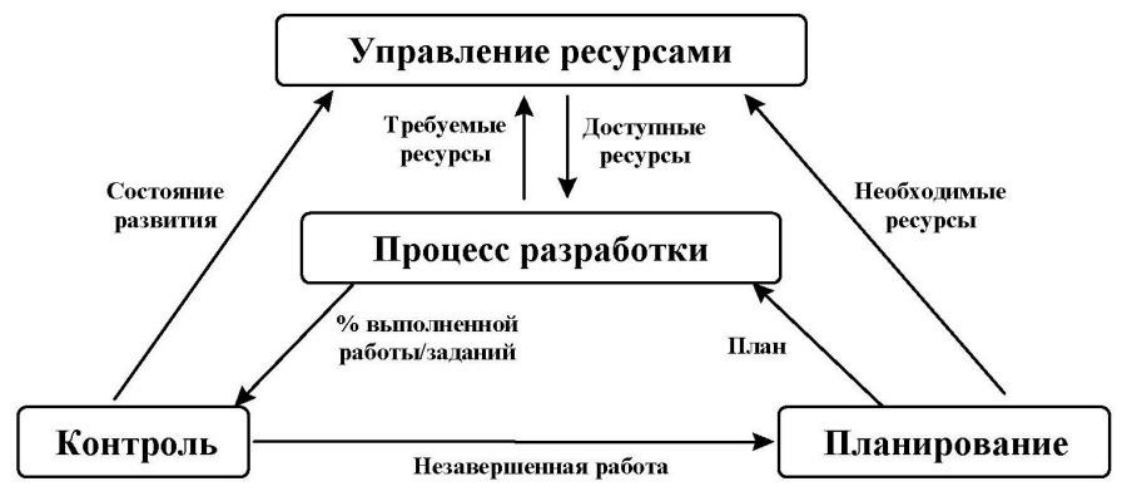

Рис. 1. Общие функциональные компоненты, взаимодействия и взаимозависимости в системе управления междисциплинарным проектом

Fig. 1. General functional components, interactions and interdependences in the framework of interdisciplinary project management 
Необходимые ресурсы определяются условиями сетевого плана развития проекта и представляют собой средства, которые нужно затратить на выполнение всего проекта, в то время как требуемые ресурсы определяются потребностями в средствах, которые нужны на некоторой стадии реализации проекта в заданный момент времени, для решения конкретной задачи. Это основное отличие между необходимыми и требуемыми ресурсами в структуре управления проектами.

\section{Базовые динамические компоненты в структуре управления проектами}

Моделирование процессов разработки и реализации междисциплинарных проектов на основе метода системной динамики осуществляется с применением специальных, но довольно общих динамических характеристик проектной и управленческой деятельности. В ряде исследований [Новиков, 2007; Привалов, 2009; Логиновский, Гельруд, 2015] предлагаются разнообразные пути описания потоков работ (заданий) в проекте, а также выделяются различные структурные элементы проектов, как например, коллектив исполнителей (кадровые ресурсы), спецификации задач и продукта, процессы доработки/переработки, доступность материальных и исполнительных ресурсов, ограничения целостности процессов, качество, размеры проекта. Системно-динамические модели описывают связи и взаимозависимости между ресурсами, процессом разработки, управлением (включая планирование), контролем исполнения проекта. Далее рассмотрим типовые динамические компоненты созданных моделей междисциплинарных проектов.

Компонент «Рабочая сила» моделирует динамику производительности, состава и компетенций коллектива исполнителей проекта при различных условиях реализации проекта на количественном и качественном уровне. Учитываются такие показатели, как мотивация, усталость, давление плана, имеющийся опыт, уровень обучаемости проектной группы и другие. Классификация и типы «Рабочей силы» предложены в исследовании [Bystrov, et. al., 2016]. Влияние суммарных компетенций и накопленного опыта проектной группы на ее производительность в ходе реализации проекта с учетом психологических и медикобиологических аспектов (мотивация, утомляемость и т. д.) формализовано в ряде моделей, предложенных, например, в работах [Путилов, Горохов, 2002; Маслобоев и др., 2015]. Известные модели управления проектами оперируют компонентом «Рабочая сила», как основным показателем ресурсных затрат и издержек в проектах, влияющих на процесс и качество выполнения проекта. Компонент «Рабочая сила» в этих моделях формализует организационные механизмы управления проектом и основные процессы распределения исполнительных ресурсов на различных фазах жизненного цикла проекта.

Компонент «Планирование» моделирует влияние принимаемых менеджерами проекта управленческих решений по изменению (уточнению) структуры проекта и путей его реализации на текущий сетевой план развития проекта посредством корректировки контрольных сроков выполнения проекта в ответ на давление текущего плана. Компонент «Планирование» используется во многих моделях, например, предложенных в [Путилов, Горохов, 2002; Новиков, 2007; Привалов, 2009]. Компонент «Планирование» описывает также динамику изменений сетевого плана проекта в соответствии с условиями реализации проекта, ожидаемыми результатами и сроками завершения работ. Уточнение или изменение контрольных сроков, как правило, приводит к снижению давления плана на ход и эффективность выполнения проекта. Это обеспечивает «люфт» и возможность доопределения плана работ по проекту, объемов требуемых ресурсов и затрат, а также позволяет снизить степень напряженности внутри проектной группы. Необходимость внесения корректировок в план реализации проекта и изменения сроков контроля этапов его выполнения на практике определяется различными факторами, к которым относятся готовность менеджеров включать новых исполнителей в проект на различных его стадиях, установленный максимально допустимый срок завершения работ по проекту и другие. 
Компонент «Реинжиниринг» моделирует процессы распознавания и корректировки ошибок в структуре управления проектом для оптимизации плана реализации проекта и улучшения требуемых показателей качества и ожидаемых результатов. Реинжиниринг является составной частью процесса реализации любого проекта, а имитирующий его компонент присутствует практически во всех известных динамических моделях проекта. Тем не менее объем ресурсов и трудозатрат на реинжиниринг проекта варьируется между различными типами проектов. Компонент «Реинжиниринг» описывает дополнительные временные и материальные затраты, необходимые для улучшения качества проекта, либо отдельных его составляющих до стадии завершения. Упрощенные динамические модели проектного реинжиниринга широко используются в моделях управления проектами как на оперативном, так и стратегическом уровнях планирования и управления. Примеры таких моделей представлены, например, в работе [Быстров, Маслобоев, 2018]. Моделирование реинжиниринга с помощью этих моделей позволяет отследить динамику возникновения типовых ошибок и оценить возможные риски (последствия) в ходе реализации проекта. Обнаружение ранее неопознанных или неявных ошибок и их своевременная обработка являются важным этапом процесса управления проектом, что расширяет понимание того, как в перспективе должен развиваться проект. Задержки своевременного распознавания и исправления ошибок в ходе планирования и реализации проекта во многом зависят от типа и масштаба проекта, а также напрямую влияют на оперативность выполнения и пути развития проекта.

Компонент «Объем работ/задач» моделирует один из определяющих показателей успешной реализации любого проекта - количество заданий, доступных для обработки в заданный момент времени на определенной стадии проекта при известном количестве уже решенных задач. Объем работ/задач также варьируется в зависимости от типа и размеров проекта и, как правило, определяется внешними и внутренними ограничениями, установленными планом реализации проекта. Накладываемые ограничения затрагивают все этапы жизненного цикла проекта, то есть результаты, полученные в ходе выполнения задач проекта на каждом этапе, определяют доступность и возможность решения задач на последующих, в том числе и предшествующих этапах реализации проекта. Последнее определяется как внешние ограничения относительно текущего этапа развития проекта.

Компонент «Объем работ/задач» в моделях проекта описывает взаимозависимость между текущими и выполненными заданиями в плане декомпозиции целей и задач проекта, а также динамику изменений спецификации этих задач при различных условиях реализации проекта. Эта взаимосвязь может меняться в зависимости от типа проекта. Так, к примеру, она может быть линейной в строительных проектах, либо нелинейной, как в проектах по разработке программного обеспечения, или вообще иметь вид неограниченного процесса, как в комплексных междисциплинарных исследовательских проектах.

Компонент «Качество» моделирует организационные механизмы распознавания ошибок в ходе реализации проекта. Это приводит к появлению новых задач в сетевом плане проекта, требующих дополнительной проработки и поиска путей решения. Кроме того, этот компонент в моделях управления проектом описывает динамику возникновения новых задач в ходе обнаружения ошибок и связанных с этими задачами итеративных процессов выполнения дополнительных работ по проекту. Учет этой динамики в процессе управления проектом необходим как для выработки путей улучшения планируемых результатов, так и для соответствия определенным стандартам качества.

Компонент «Масштабирование» моделирует динамику изменений в размерах проекта при различных условиях, меняющихся на разных этапах его реализации. Причины изменений масштабов проекта зачастую связаны с возникновением новых целей и задач, доопределением сетевого плана проекта, перераспределением ресурсов, уточнением или модификацией организационных, технологических и нормативных требований к проекту, результатам его реализации или сопутствующим процессам, обнаружением ошибок и несогласованности между звеньями управляющего цикла проекта. Например, в работе [Маслобоев, Путилов, 2016] рассматривается динамическая модель оценки масштабов инновационных проектов при 
изменениях спецификаций $\quad$ в требованиях $к$ наукоемкому продукту и изменениях инвестиционного климата в регионе. Использование компонента «Масштабирование» в моделях проекта обеспечивает оценку и анализ влияния масштабов проекта на контрольные сроки его реализации, необходимый объем исполнительных ресурсов, а также на длительность жизненного цикла развития проекта.

Компонент «Потоки заданий/работ» моделирует структуру процесса реализации задач проекта и цепочек действий (потоков работ) по решению этих задач в рамках этапов жизненного цикла проекта. В различных моделях проектов данный компонент может использоваться для системного представления как полного цикла развития и реализации проекта, так и отдельных процессов и этапов жизненного цикла, являющихся наиболее существенными и критичными для конкретного типа и масштаба проекта. В моделях сложных многофазовых междисциплинарных проектов, как правило, описываются этапы постановки задач, формирования проектной группы, спецификации конечных результатов и анализа их применимости, разработки и исследования, производства или реализации, экспериментального тестирования и другие. Этапы проектирования и планирования также включаются в эти модели в виде отдельных подмоделей посредством установления связей между всеми объектами модели через функционал компонента «Потоки заданий/работ».

Рассмотренные функциональные компоненты динамических моделей управления проектами демонстрируют многоаспектность и структурную сложность управления жизненным циклом проектов различного типа и уровня. Так как эти компоненты активно взаимодействуют и интегрируются друг с другом в реальных приложениях, то сложность моделирования проектного управления и интерпретации результатов прогноза развития проектов традиционными методами в некоторой степени увеличивается. Это затрудняет управление крупномасштабными и междисциплинарными проектами. В связи с этим системно-динамические имитационные модели призваны помочь в решении задач анализа взаимосвязей между структурой управления и динамикой сложных масштабных проектов.

\section{Технология системно-динамического моделирования проектного управления}

На практике модели системной динамики широко применяются на двух основных уровнях иерархии управления проектами - стратегическом и оперативном уровне (рис. 2) [Путилов, Горохов, 2002]. Стратегический уровень управления включает основные этапы реализации проекта и охватывает весь жизненный цикл проекта от начала и до конца. Этот уровень описывается глобальной системно-динамической моделью. Оперативный (операционный) уровень управления, наоборот, содержит взаимосвязанные подпроцессы и составные части проекта, которые моделируются своей специализированной системнодинамической моделью. Этот уровень приложения системной динамики ориентирован на прогнозирование развития проекта в будущем и изменения его состояний до завершения очередного управляющего цикла при наличии доступной и достоверной информации о характеристиках этого цикла для формирования/корректировки плана реализации проекта.

Между традиционными сетевыми моделями управления проектами и моделями системной динамики выделяют два типа связей - информационные и структурные связи. Так, структура системно-динамической модели проекта строится изоморфно, то есть адекватно структуре сетевых моделей, а потоки данных (входная и выходная информация) в системнодинамической модели соответствуют принятой информационной структуре, используемой в традиционных моделях управления проектами. Это позволяет обеспечить имитацию динамики поведения проекта посредством системно-динамической модели, хорошо согласующуюся с результатами применения аналогичной сетевой модели проекта. Логическая интеграция формальных сетевых моделей и моделей системной динамики на стратегическом и оперативном уровнях управления проектом обеспечивают, в свою очередь, установление и поддержку аналитических связей между этими типами моделей в процессе анализа и моделирования жизненного цикла проекта и проектного менеджмента. 


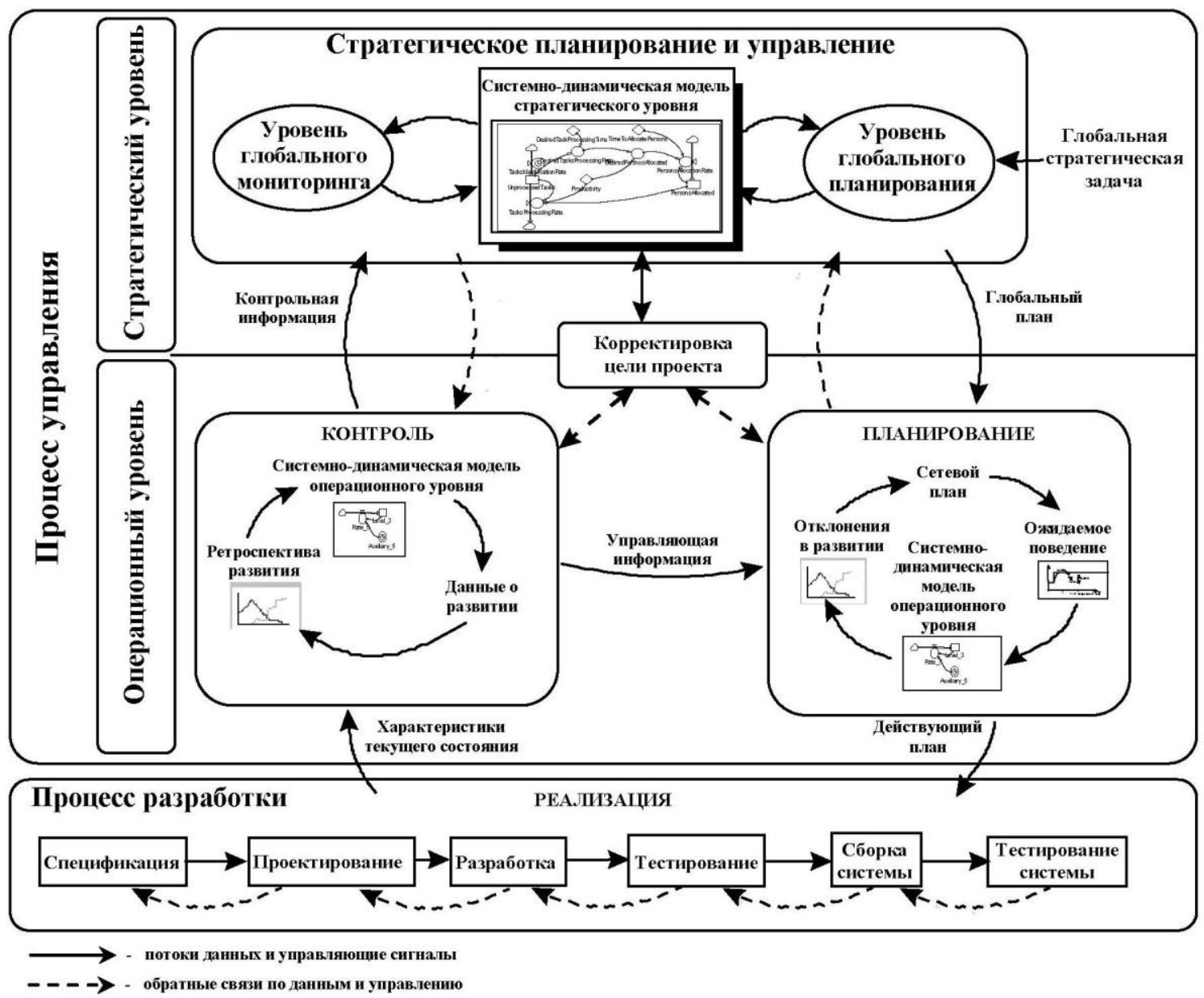

Рис. 2. Двухуровневая структура применения системно-динамических моделей в управлении проектами

Fig. 2. Two-level structure of system dynamic model application in the project management

На этапе планирования в структуре управления проектом системно-динамические модели используются в следующих целях:

1) Для распознавания метрических характеристик процесса развития проекта, определенных в исходном или текущем сетевом плане реализации проекта и динамически меняющихся со временем. Эти динамические параметры, описывающие метрику проекта, характеризуют планируемые затраты на поэтапное выполнение проекта, эффективность работы, производительность исполнительных ресурсов, пространственно-временные и ресурсные ограничения на управление проектом и т. д. По данным характеристикам оценивается реалистичность сетевого плана проекта.

2) Для анализа жизнеспособности сетевого плана проекта в условиях влияния различных факторов риска - внешних и внутренних, с прицелом на перспективу.

3) Для определения альтернативных и выбора наилучших путей достижения целей реализации проекта и требуемых показателей качества управления проектом при заданных условиях на основе оценки альтернативных вариантов решений по разработке наукоемкого продукта и анализа сценариев проектного управления при распределении и планировании исполнительных и других необходимых видов ресурсов.

4) Для идентификации возможных коллизий и флуктуаций в сетевом плане развития проекта на основе прогнозирования динамики показателей результативности проекта как на оперативном, так и стратегическом уровне управления.

На этапах мониторинга и контроля исполнения в структуре управления проектом системно-динамические модели применяются по следующим направлениям: 
1) Анализ неопределенности и риска развития проекта на основе распознавания косвенной или неявной фактологической информации о текущем состоянии реализации проекта.

2) Сравнительный анализ и оценка исходных и фактических метрических свойств процесса развития проекта и установление достигнутых результатов его реализации в заданный момент времени.

3) Определение возможностей улучшения результатов реализации проекта на конкретном этапе цикла его развития путем оптимизации механизмов организационного управления проектом на основе исследования альтернативных вариантов управленческих решений, принимаемых при планировании и распределении ресурсов на выполнение проекта, структурировании и декомпозиции проекта, выборе управляющих воздействий на элементы и процессы жизненного цикла проекта и т. д.

В том и другом приложениях системно-динамические модели обеспечивают функциональное расширение системы управления проектами за счет предоставления возможностей имитации, предсказания и обоснования характера поведения проекта и его динамических характеристик в тех или иных условиях.

Обобщение путей использования системно-динамических моделей проекта в цикле управления проектом представлено на рис. 3.

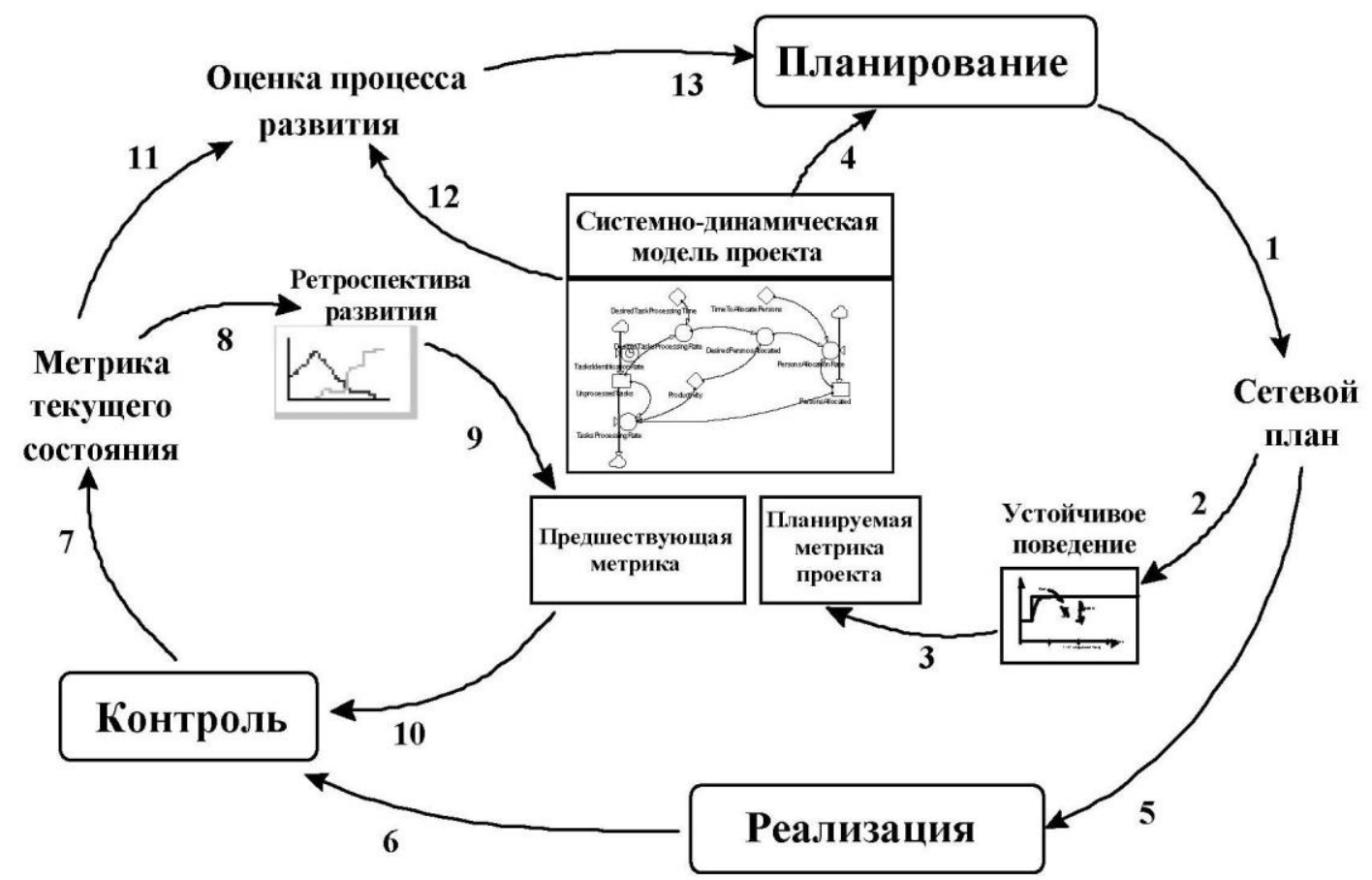

Рис. 3. Схема технологии применения системной динамики в управлении проектами

Fig. 3. Technological scheme of system dynamics application in project management

Как показано на рис. 3, технология применения системно-динамических моделей в задачах управления междисциплинарными проектами реализуется в несколько шагов:

Шаг 1. Разработка, доопределение или корректировка параметров сетевого плана для очередного управляющего цикла проекта.

Шаг 2. Формирование (прогноз) сценария устойчивого поведения функциональных элементов проекта, определенного к сетевому планированию.

Шаг 3. Калибрование метрики процесса развития и реализации проекта в системнодинамической модели проекта, обеспечивающее имитацию запланированного поведения проекта на модели.

Шаг 4. Выявление нестыковок и коллизий в исходном и желаемом сетевом плане проекта на основе анализа откалиброванной метрики процесса с применением системно- 
динамической модели проекта, что позволяет предварительно оценить возможные риски реализации отдельных этапов проекта и эффекты от принятия альтернативных вариантов решений на стадии планирования проекта.

Шаг 5. Реализация модифицированного сетевого плана проекта в ходе выполнения очередного управляющего цикла проекта.

Шаг 6. Сбор информации и мониторинг состояния развития проекта и процесса его реализации в ходе основных этапов жизненного цикла проекта.

Шаг 7. Формирование аналитической информации о значениях метрики процесса развития проекта и подготовка периодической отчетности.

Шаг 8. Построение набора взаимосвязанных ретроспективных данных о значениях метрических характеристик процесса развития проекта.

Шаг 9. Калибрование метрики процесса реализации проекта с помощью системнодинамической модели проекта, обеспечивающее имитацию ретроспективы всего цикла развития проекта на модели.

Шаг 10. Выявление несоответствий в собранной информации в ходе калибрования метрики процесса реализации проекта, что обеспечивает возможность получения дополнительных данных о развитии проекта, которые используются для расширения сведений о текущих метрических характеристиках, на основе откалиброванной системно-динамической модели.

Шаг 11. Формирование сводного отчета о значениях метрических характеристик процесса развития проекта на стадии промежуточного контроля хода исполнения проекта.

Шаг 12. Сопоставление запланированных на шаге 3 и фактических метрических характеристик процесса развития проекта с применением системно-динамической модели проекта, что позволяет обнаружить отклонения в процессе управления проектом и показателях его эффективности, а также исследовать альтернативные пути достижения наилучших результатов.

Шаг 13. Калибровка системно-динамической модели проекта с целью имитации предшествующих этапов управляющего цикла проекта, что позволяет спрогнозировать будущее состояние проекта, которое может отличаться от исходного или текущего (скорректированного) сетевого плана проекта.

Шаг 14. Модификация и обновление текущего сетевого плана реализации проекта для следующего управляющего цикла на основе анализа информации, полученной на шаге 13.

Представленная последовательность шагов является технологической схемой для практического использования метода системной динамики и имитационных моделей в информационно-аналитической поддержке управления междисциплинарными проектами.

\section{Заключение}

В работе дано научное обоснование применения системной динамики для анализа и моделирования процессов управления комплексными междисциплинарными проектами. Разработана технологическая схема (технология) использования системно-динамических моделей в процессе управления междисциплинарными проектами, обеспечивающая концептуальный анализ динамической структуры проектов и моделирование динамики их поведения в условиях временных и ресурсных ограничений. На концептуальном уровне технология определяет правила того, как системно-динамические модели интегрируются в общие модели управления проектами и как это может быть использовано для контроля и планирования проектной деятельности, а также для достижения заданных показателей эффективности реализации и управления проектами. Результаты работы смогут найти применение для улучшения известных отечественных и зарубежных практик управления проектами в научно-инновационной, образовательной, инвестиционной и других сферах.

В ходе дальнейших исследований планируется установить четкую схему для таких критических вопросов, как адекватность модели, общность, «стандартность» структуры 
модели и процесса разработки, автоматизация калибровки модели, а также разработка стандартной метрики для моделирования процессов управления междисциплинарными проектами. Определенный интерес представляет попытка объединения концептуальных и системно-динамических моделей в единую метамодель управления междисциплинарными проектами, что может служить фундаментальной основой для развития теории и практики управления проектами различного уровня в сфере научных исследований и разработок.

Результаты исследования были использованы при реализации Стратегии развития Арктической зоны Российской Федерации и обеспечения национальной безопасности на период до 2035 года (утверждена Указом Президента РФ от 26 октября 2020 года № 645) в части решения задач по оптимизации временных, материальных и финансовых затрат на планирование, реализацию и координацию социально-направленных междисциплинарных научно-исследовательских проектов на территории Мурманской области.

\section{Список литературы}

1. Быстров В.В., Маслобоев А.В. 2018. Концептуальная модель жизненного цикла управления проектами в сфере обеспечения региональной безопасности. Информационные системы и технологии, 3 (107): 48-56.

2. Игнатьев М.Б., Путилов В.А., Смольков Г.Я. 1986. Модели и системы управления комплексными экспериментальными исследованиями. М., Наука, 228.

3. Кузьмин И.А., Путилов В.А., Фильчаков В.В. 1991. Распределенная обработка информации в научных исследованиях. Л., Наука, 304.

4. Логиновский О.В., Гельруд Я.Д. 2015. Информационно-аналитическая система управления проектами на базе использования комплекса математических моделей функционирования стейкхолдеров. Вестник ЮУрГУ. Серия «Компьютерные технологии, управление, радиоэлектроника», 15 (3): $133-141$.

5. Маслобоев А.В. 2019. Концепция Центра перспективных исследований и обеспечения безопасности Арктики. Арктика: экология и экономика, 2 (34): 129-143.

6. Маслобоев А.В., Олейник А.Г., Шишаев М.Г. 2015. Информационная технология дистанционного формирования и управления моделями системной динамики. Научно-технический вестник информационных технологий, механики и оптики, 15 (4): 748-755.

7. Маслобоев А.В., Путилов В.А. 2016. Информационное измерение региональной безопасности в Арктике. Апатиты, КНЦ РАН, 222.

8. Медоуз Д.Х., Рандерс Й., Медоуз Д.Л. 2007. Пределы роста. 30 лет спустя. М., ИКЦ «Академкнига», 342.

9. Новиков Д.А. 2007. Управление проектами: организационные механизмы. М., ПМСОФТ, 140.

10. Олейник А.Г., Ломов П.А. 2016. Разработка онтологии интегрированного пространства знаний. Онтология проектирования, т. 6, 4 (22): 465-474.

11. Олейник А.Г., Фридман А.Я. 2020. Структура комплексных концептуальных пространств в междисциплинарных проектах. Труды Кольского научного центра РАН, т. 11, 8 (11): 150-154.

12. Привалов А.И. 2009. Математические модели управления проектами в решении системных проблем экономики. Сегодня и завтра российской экономики, 26: 43-48.

13. Путилов В.А., Горохов А.В. 2002. Системная динамика регионального развития. Мурманск, НИЦ «Пазори», 306.

14. Bystrov V.V., Malygina S.N., Khaliullina D.N. 2016. The information technology of multi-model forecasting of the regional comprehensive security. Advances in Intelligent Systems and Computing, vol. 466, 3: $475-482$.

15. Forrester J.W. 2007a. System dynamics - a personal view of the first fifty years. System Dynamics Review, vol. 23, 2-3: 345-358.

16. Forrester J.W. 2007b. System dynamics - the next fifty years. System Dynamics Review, 23, 2-3: 359-370.

\section{References}

1. Bystrov V.V., Malygina S.N., Khaliullina D.N. 2016. The information technology of multi-model forecasting of the regional comprehensive security. Advances in Intelligent Systems and Computing, 466, 3: 475-482. 
2. Bystrov V.V., Masloboev A.V. 2018. Conceptual model of the project management life-cycle in the field of regional security support. Information systems and technologies, 3 (107): 48-56. (in Russian)

3. Forrester J.W. 2007a. System dynamics - a personal view of the first fifty years. System Dynamics Review, vol. 23, 2-3: 345-358. $359-370$.

4. Forrester J.W. 2007b. System dynamics - the next fifty years. System Dynamics Review, 23, 2-3:

5. Ignatyev M.B., Putilov V.A., Smol'kov G.Ya. 1986. Management models and systems of the comprehensive experimental research. Moscow, Nauka, 228. (in Russian)

6. Kuz'min I.A., Putilov V.A., Fil'chakov V.V. 1991. Distributed processing of information in scientific research. Leningrad, Nauka, 304. (in Russian)

7. Loginovskiy O.V., Gelrud Ya.D. 2015. Informational and analytical system of project management on the basis of a complex of mathematical models of the functioning of stakeholders. Bulletin of the SUSU. Series "Computer technologies, control, radio electronics", vol. 15, 3: 133-141. (in Russian)

8. Masloboev A.V. 2019, The Concept of the Arctic Center for Advanced Research and Security Support. Arctic: ecology and economy, 2(34): 129-143. (in Russian)

9. Masloboev A.V., Oleynik A.G., Shishaev M.G. 2015. Remote synthesis and control information technology of system-dynamic models. Scientific-technical Journal of information technologies, mechanics and optics, vol. 15, 4: 748-755. (in Russian)

10. Masloboev A.V., Putilov V.A. 2016. Information dimension of regional security in the Arctic. Apatity: KSC RAS, 222. (in Russian)

11. Medouz D.Kh., Randers Y., Medouz D.L. 2007. Growth limits. 30 years later. Moscow: IKTs «Akademkniga», 342. (in Russian) (in Russian)

12. Novikov D.A. 2007. Project management: organizational mechanisms. Moscow: PMSOFT, 140.

13. Oleynik A.G., Lomov P.A. 2016. Development of the ontology of integrated knowledge space. Ontology design, vol. 6, 4(22): 465-474. (in Russian)

14. Oleynik A.G., Fridman A.Ya. 2020. Structure of integrated conceptual spaces in interdisciplinary projects. Bulletin of the Kola science center RAS, vol. 11, 8(11): 150-154. (in Russian)

15. Privalov A.I. 2009. Mathematical models of project management in solving system problems of the economy. Today and Tomorrow of the Russian Economy, 26: 43-48. (in Russian)

16. Putilov V.A., Gorokhov A.V. 2002. System dynamics of regional development. Murmansk: NITs «Pazori», 306. (in Russian)

Конфликт интересов: о потенциальном конфликте интересов не сообщалось.

Conflict of interest: no potential conflict of interest related to this article was reported.

\section{ИНФОРМАЦИЯ ОБ АВТОРАХ}

Маслобоев Андрей Владимирович, доктор технических наук, доцент, ведущий научный сотрудник лаборатории информационных технологий управления региональным развитием, Институт информатики и математического моделирования Федерального исследовательского центра «Кольский научный центр Российской академии наук», г. Апатиты, Россия

Путилов Владимир Александрович, доктор технических наук, профессор, научный руководитель, Институт информатики и математического моделирования Федерального исследовательского центра «Кольский научный центр Российской академии наук», г. Апатиты, Россия

\section{INFORMATION ABOUT THE AUTHORS}

Andrey V. Masloboev, Doctor of Technical Sciences, Associate Professor, Leading Researcher in Department of information technologies for regional development management, Institute for Informatics and Mathematical Modeling of the Federal Research Center "Kola Science Center of the Russian Academy of Sciences", Apatity, Russia

Vladimir A. Putilov, Doctor of Technical Sciences, Professor, Scientific director, Department of information technologies for regional development management, Institute for Informatics and Mathematical Modeling of the Federal Research Center "Kola Science Center of the Russian Academy of Sciences", Apatity, Russia 